\title{
Programa de Tutorías Académicas en Competencias de Lectura y Escritura: su enfoque pedagógico ${ }^{1}$
}

\author{
Mariana Urús \\ Universidad Tecnológica Nacional, \\ Facultad Regional Buenos Aires \\ marianaurus@gmail.com
}

Recibido: 15-05-2018

Aprobado: 24-12-2018

1. El presente trabajo fue realizado en el marco del Proyecto de Investigación "Tutoría universitaria y políticas de inclusión: análisis de configuraciones e impactos desde perspectivas institucionales, nacionales y latinoamericanas" en la Facultad Regional Buenos Aires, Universidad Tecnológica Nacional (Argentina). 


\title{
Programa de Tutorías Académicas en Competencias de Lectura y Escritura: su enfoque pedagógico
}

\begin{abstract}
Resumen
La enseñanza de la lectura y la escritura en la educación superior implica prácticas pedagógicas vinculadas con las culturas institucionales y las concepciones compartidas por sus miembros, que no siempre son explícitas. En la actualidad, la alfabetización académica cobra relevancia como práctica incorporada a los distintos campos disciplinares con la finalidad de promover mejores aprendizajes integrando la lectura y la escritura como actividades de las cátedras universitarias. En este encuadre, se presenta un trabajo de investigación sobre el Programa de Tutorías Académicas en Competencias de Lectura y Escritura, que se implementa en la Facultad Regional General Pacheco de la Universidad Tecnológica Nacional (Argentina). El propósito es aportar a la comprensión del enfoque pedagógico del programa a partir de la perspectiva de los principales actores que lo implementan y teniendo en cuenta las siguientes dimensiones de análisis: los problemas a los que busca responder el proyecto, los significados atribuidos a la relación entre los saberes a enseñar y la escritura académica, la construcción del vínculo didáctico entre docentes y tutores, las fortalezas y las debilidades del programa, y los cambios impulsados a futuro. La metodología utilizada es cualitativa y de carácter exploratorio. Se presentan, además, contribuciones y líneas propositivas para enriquecer el enfoque pedagógico actual.

Palabras clave: enseñanza superior, tutoría, enseñanza de la escritura, ingeniería, pedagogía
\end{abstract}

\section{Program of Academic Tutoring in Reading and Writing Competences: the pedagogical approach}

\section{Abstract}

The teaching of reading and writing in Higher Education implies pedagogical practices linked to shared institutional cultures and views, which are not always explicit. Currently, academic reading and writing become relevant as practices incorporated into the different disciplinary fields in order to promote better learning: integrating reading and writing as activities within university chairs. This article presents research results on a program of academic tutoring in reading and writing competences which is implemented in a specific institutional context (Regional School General Pacheco at the Universidad Tecnológica Nacional, Argentina). This research aims to contribute to the understanding of the program's pedagogical approach from the perspective of the main agents implementing it, taking into account the following analytical dimensions: issues addressed by the program, meanings attributed to the relationship between knowledge to be delivered and academic writing, the construction of the didactic link between teachers and tutors, the strengths and weaknesses of the program and future-driven changes. The methodology used is qualitative and exploratory. In addition, this work presents contributions and proposals lines to enrich the current pedagogical approach.

6 I Keywords: Higher Education, tutoring, academic writing, Engineering, pedagogy 


\section{Introducción}

El presente trabajo aborda como tema central los resultados de una investigación sobre el enfoque pedagógico del Programa de Tutorías Académicas en Competencias de Lectura y Escritura - en adelante, Programa de Tutoríasque se implementa en la Facultad Regional General Pacheco de la Universidad Tecnológica Nacional (Argentina) desde el año 2007 en un conjunto de asignaturas de las carreras de Ingeniería Eléctrica e Ingeniería Mecánica. El objetivo fue explorar las características que adquieren los diferentes aspectos que integran el enfoque didáctico del programa con la finalidad de brindar aportes al mismo.

El desarrollo de competencias de lectura y escritura en la universidad implica prácticas vinculadas con las culturas institucionales y las concepciones compartidas por sus miembros, no siempre de modo explícito. Al mismo tiempo, pone en tensión aspectos de la didáctica universitaria con respecto a la forma de llevar adelante esas prácticas y a quién debe responsabilizarse de ello. En este marco, el interés de este estudio radica en la modalidad de las intervenciones didácticas que se realizan desde el Programa de Tutorías, en el que intervienen especialistas en competencias comunicativas con docentes de carreras de Ingeniería.

El Programa se desarrolla dentro de las cátedras de las materias integradoras $^{2}$ de los dos primeros años. El objetivo principal es fortalecer los saberes discursivos de los estudiantes. Además, se propone que dicha intervención movilice la reflexión sobre la práctica de escritura que propician los docentes universitarios para modificar las propuestas didácticas empleadas en la formación de los estudiantes. Este objetivo se sostiene a partir de la noción de alfabetización académica de Carlino (2005), quien legitima la enseñanza y la práctica de los procesos de lectura y escritura inherentes a cada cátedra universitaria en su seno, debido a que cada disciplina aborda prácticas del lenguaje específicas que deben ser compartidas por profesores y estudiantes. Siguiendo esa línea, la peculiaridad de las materias integradoras habilita la labor tutorial realizada desde el programa descrito.

Para llevar adelante el Programa de Tutorías, se constituyó una pareja interdisciplinaria de trabajo que colabora con el docente responsable de la materia y está integrada por un docente de Ingeniería y un docente del área de Lengua (Laco y Guiggiani, 2008). El docente de Lengua coordina su acción en dos direcciones simultáneamente: por un lado, con el docente ingeniero y, por otro, con los estudiantes a través de intervenciones grupales.

2. La denominación "materia integradora" constituye un elemento distintivo de los diseños curriculares vigentes en la UTN. Su función consiste en vincular horizontal y verticalmente las otras asignaturas a través de contenidos y actividades que pongan al estudiante en situaciones similares a las que la vida profesional le propondrán (Laco y Guiggiani, 2008). 
De acuerdo con el tipo de actividad propuesto por la cátedra, y utilizando los insumos textuales de la misma, el tutor desarrolla y ejercita estrategias que resulten productivas para los objetivos planteados por las asignaturas. El estudiante como usuario novicio de modalidades académicas de la lengua escrita requiere decisivamente de un apoyo y una retroalimentación de los tutores como conocedores de la comunicación escrita en su disciplina, capaces de apoyar el desarrollo de las habilidades que corresponden a su manejo (Laco y Guiggiani, 2008).

El objetivo general de este trabajo de investigación es analizar, desde la perspectiva de los docentes involucrados, los principales aspectos que integran el enfoque pedagógico del Programa de Tutorías Académicas en Competencias de Lectura y Escritura con la finalidad de contribuir a su comprensión y mejora. De manera concreta, los objetivos han implicado tres instancias. En primer lugar, ha supuesto describir el vínculo que se establece entre los docentes universitarios y los tutores en competencias de lectura y escritura. En segundo lugar, se han identificado las fortalezas y las debilidades del proyecto didáctico del programa, y las propuestas de mejora planteadas por los actores participantes. Finalmente, ha implicado identificar los cambios impulsados por el programa en lo que respecta a los aprendizajes de los estudiantes, las prácticas de enseñanza de los docentes universitarios y las prácticas de escritura de los estudiantes, así como elaborar líneas propositivas para el enfoque pedagógico.

El aporte de esta investigación radica en que procura enriquecer el complejo entramado de una didáctica sociocultural de la lengua en el ámbito universitario (Bixio, 2003; Bombini, 2005).

\section{Antecedentes}

En relación con la enseñanza de las prácticas de lectura y escritura en el ámbito universitario aparecen como antecedentes, por un lado, los aportes de autores como Desinano (2009), Litwin (1997) y Mercer (1997), quienes revalorizan la utilización del lenguaje en las prácticas áulicas, y, por otro lado, las nociones: escritura académica propuesta por Carlino $(2005,2013)$ y literacidad académica difundida por Zavala (2009). Desde estas perspectivas, la escritura académica se aborda como una estrategia de enseñanza que implica considerar su relación con el saber que tanto docentes como estudiantes han construido mediante las prácticas de escritura y los aspectos epistémicos e identitarios que se ponen en juego dentro de ellas. Para comprender las relaciones con el saber que los sujetos construyen, Charlot (2008) conceptualiza y desarrolla la noción relación con el saber y expone los distintos tipos de relaciones que ha identificado.

Cabe mencionar que la lectura y la escritura en la educación superior ha sido tema central en numerosos encuentros realizados en distintas ciudades americanas: Writing Research Across Borders (Santa Bárbara, 2008), XII Congreso Latinoamericano para el Desarrollo de la Lectura y la Escritura (Puebla, 8 I 2013), IV Foro Iberoamericano de Literacidad y Aprendizaje (Puebla, 2013), 
Writing Research Across Borders (Bogotá, 2017). A partir del análisis de los ejes temáticos y las publicaciones realizadas en estos encuentros, se identifican narrativas con diversas propuestas de intervención, en las que prevalecen cursos, talleres o ayudas externas a las cátedras universitarias. De manera aislada, se observan experiencias de enseñanza en el interior de las asignaturas, el plan de estudios o cátedras compartidas por profesionales de diferentes áreas (Elissetche, 2010; Estienne, 2012; Garbarini, Escobar, Lavigna y López D’ Amato, 2016; Murillo, 2012; Natale y Stagnaro, 2013; Zerillo, 2014).

La investigación en torno a la temática de la tutoría, sus sentidos, motivaciones y modelos, tiene antecedentes en Argentina. No obstante, aún es insuficiente el análisis teórico complejo de sus relaciones con la enseñanza de prácticas letradas. Solo se identifica algunos trabajos presentados en jornadas, en las que aparecen distintas propuestas de enseñanza de escritura académica dentro de un espacio denominado tutoría (Baldoni et. al., 2005; Castronovo y Mancovsky, 2010; Díaz, Stangatti y Festa, 2012; García, Nícoli y Urús, 2012; Rigonat y García, 2012; Urús, 2017).

\section{Marco de referencia}

El enfoque didáctico del Programa de Tutorías se enmarca en distintos enfoques teóricos. Un primer aporte proviene de la didáctica sociocultural de la lengua. Esta configuración disciplinar ha sido propuesta por Gustavo Bombini, quien sostiene que se trata de una perspectiva interdisciplinaria, pues "parte de la tarea de circunscribir problemas y formular hipótesis para cuya comprensión es necesario apelar al uso de paradigmas posicionados en la reflexión pedagógica, sociológica, cultural e histórica” (Bombini y Cuesta, 2006, p. 6). La mirada investigativa desde esta perspectiva entiende que no se puede despojar a la lengua - en tanto objeto de enseñanza- de los aspectos históricos, sociales y culturales que la complejizan.

Dentro de las prácticas de enseñanza, el lenguaje tiene un lugar protagónico, porque es el instrumento con el cual el docente habilita u obstaculiza la construcción del conocimiento por parte de los estudiantes. El lenguaje dentro del contexto de aula se convierte en una dimensión para analizar desde diferentes aristas: la teoría del déficit y la teoría de la diferencia (dos miradas desde la "carencia lingüística" de los estudiantes), la construcción guiada del conocimiento a través del lenguaje (Cazden, 1991; Mercer, 1997) y la teoría de la acción comunicativa que expone Litwin (1996).

Desde la psicología sociocultural, Mercer (1997) expone que el conocimiento es una posesión conjunta, que se construye socialmente con los otros; en esa construcción, "el lenguaje se concibe como una forma social de pensamiento" (p. 4). Las interacciones lingüísticas entre docentes y estudiantes promueven en el aula un contexto de aprendizaje, donde una comunidad de aprendices va transitando por una apropiación guiada de significados a través de las negociaciones que propician las prácticas conversacionales. Desde esta 
perspectiva, Litwin (1996) propone que una teoría de la acción comunicativa debería analizar de manera crítica la función lingüística en el aula para poder interpretar los problemas del discurso pedagógico. Además, sostiene que el docente podrá negociar significados siempre que sepa por qué el estudiante plantea lo que plantea, y pueda reconocer el origen de sus afirmaciones y hacia dónde se dirige su pensamiento.

De esta forma, las prácticas conversacionales - orales y escritas- en tanto devoluciones de los profesores configuran el marco de una red distribuida de conocimiento especializado. Tanto Rogoff (1993) como Baquero (2001) desarrollaron aportes sobre comunidades de aprendizaje y enseñanza recíproca y colaborativa, en las que las interacciones lingüísticas son fundamentales. Estos autores consideran que el aprendizaje implica una acción situada y distribuida, puesto que el conocimiento y la adquisición del conocimiento tienen una naturaleza social y cultural.

Otro aporte disciplinar es el concepto de alfabetización académica (Carlino, 2005). Esta noción legitima la enseñanza y la práctica de los procesos de lectura y escritura inherentes a cada cátedra universitaria en su seno, pues cada disciplina aborda prácticas del lenguaje específicas que deben ser compartidas por estudiantes y profesores. De esa manera, se acompaña a los jóvenes en la mejora de sus competencias comunicativas para que puedan desenvolverse adecuadamente como usuarios de la lengua dentro del ámbito universitario y laboral. En 2005, Carlino publica argumentos sobre la importancia de la alfabetización académica tomando como base el potencial cognitivo de la escritura, y las experiencias desarrolladas en Norteamérica y Australia. No obstante, en 2013, revisa la primera concepción y sostiene que la noción se definía en torno a las habilidades que adquirían y a los procesos que atravesaban los estudiantes al transitar por la universidad, y no se centraba en el trabajo que los docentes podían realizar para lograr que sus estudiantes desarrollen dichas habilidades (Carlino, 2013). En función de esto, plantea una nueva definición de alfabetización académica: "Proceso de enseñanza que puede (o no) ponerse en marcha para favorecer el acceso de los estudiantes a las diferentes culturas escritas de las disciplinas" (Carlino, 2013, p. 370).

Por otra parte, Virginia Zavala explica la noción de literacidad académica que implica "saber cómo hablar y actuar en Discursos académicos" (2009, p. 349). La autora entiende que la literacidad no puede enseñarse de manera formal en algunas clases y afirma: "la gente se vuelve letrada observando e interactuando con otros miembros del Discurso hasta que las formas de hablar, actuar, pensar, sentir y valorar comunes a ese Discurso se vuelven naturales a ellos" (Zavala, 2009, p. 349).

Una contribución valiosa que se ha adoptado es la noción de relación con el saber, analizada por Charlot (2008). La actividad cognitiva que se construye a través de las prácticas educativas implica un conjunto de conocimientos, afectos, motivaciones acerca de algo que relaciona al ser humano con el mundo. Charlot aborda esas relaciones que el sujeto establece con lo que lo 
rodea y afirma que el saber es relación. Abordar la relación con el saber implica estudiar las interacciones que el sujeto establece con lugares, personas, objetos, contenidos de pensamiento, situaciones, normas relacionales, puesto que estas se articulan entre sí en configuraciones que el autor denomina "figuras del aprender". Entre dichas configuraciones, encuentra tres formas de relación epistémica con el saber. La primera, llamada "objetivación-denominación", constituye un proceso en el cual aprender implica pasar de la identificación de un saber virtual a su apropiación real y en que el sujeto es consciente de haberse apropiado de ese saber. En este caso, el lenguaje, sobre todo escrito, desempeña un papel fundamental, porque permitiría dar cuenta de que se produjo la apropiación real de ese objeto. La segunda se denomina "imbricación del Yo en situación". Se trata de aprender a dominar una actividad emprendida en el mundo, de forma pertinente. La tercera figura del aprender es presentada como "distanciamiento-regulación". Según el autor, el sujeto epistémico es el sujeto afectivo y relacional que es capaz de regular esa relación, y de encontrar la distancia entre sí mismo y los otros -en situación-.

Cuando los docentes universitarios se refieren a la relación con el saber sobre las prácticas de escritura de los estudiantes, es frecuente que consideren y afirmen que dichas prácticas debieron ser adquiridas en los estadios educativos anteriores. Carlino (2005) expone que estas ideas y representaciones de los docentes se reflejan en las propuestas didácticas que les plantean a sus estudiantes para que se apropien de los contenidos específicos del área. Debido a ello, muchas veces, se proponen actividades que no suelen atender a las dificultades a las que se enfrentan los estudiantes al momento de abordar y apropiarse de los contenidos de las cátedras. Por eso, es importante considerar la escritura académica como una estrategia de enseñanza, sin dejar de tener en cuenta las reflexiones que Bombini (2009) plantea al respecto. El autor cuestiona el hecho de focalizar la mirada en los géneros discursivos académicos como si se tratase una "solución mágica" y como si se pudiera escribir con solo conocer las características de cada uno de ellos. Explica que la propia definición de los llamados géneros académicos es controvertida, pues no se pueden comparar los textos producidos en una situación de escolarización con los elaborados en la vida profesional.

Otros aportes teóricos que enriquecen el análisis del enfoque didáctico del programa son los de Litwin (1997), quien afirma que la configuración didáctica es "la manera particular que despliega el docente para favorecer los procesos de construcción del conocimiento" (p. 97). En esa forma particular, se puede reconocer cómo el docente concibe las temáticas de su campo disciplinar y cómo las organiza; los supuestos sobre el aprendizaje que se ponen en juego en sus clases; los vínculos que establece entre su disciplina y las prácticas profesionales; el estilo de negociación de significados que genera, y las relaciones que surgen entre la práctica y la teoría - y que incluyen lo metódico, y la relación entre el saber y el ignorar-.

Litwin (1996) reflexiona sobre las prácticas de enseñanza y distingue la buena enseñanza y la enseñanza comprensiva. Para describir la buena 
enseñanza, recurre a Fenstermacher (1989). Según este autor, la enseñanza implica proporcionar a los estudiantes una serie adecuada de oportunidades para que tengan acceso al contenido. En este contexto, la buena enseñanza se da cuando el accionar docente puede justificarse basándose en principios morales, y cuando los contenidos que se enseñan son racionalmente justificables y dignos de ser apropiados por los estudiantes. Por su parte, para que la enseñanza sea comprensiva, debería favorecer "el desarrollo de procesos reflexivos, el reconocimiento de analogías y contradicciones y permanentemente recurrir al nivel de análisis epistemológico" (Litwin, 1996, p. 97). En definitiva, para que la buena enseñanza se imbrique con la enseñanza comprensiva, habría que encontrar para cada contenido la mejor manera de enseñarlo. Esto evitaría caer en la utilización de métodos generales, ajenos a la especificidad de los contenidos disciplinares.

En esta línea, y desde el enfoque sociocultural, el concepto de educabilidad es clave para el análisis del programa y de su relación con el concepto de aprendizaje. Por ello, se adhiere a los aportes de Baquero (2006) y de Gutiérrez y Rogoff (2003), quienes conciben el aprendizaje como un proceso enmarcado por una actividad, en el cual los sujetos pueden participar de diversos modos en actividades conjuntas. En esa medida, en el centro del proceso de aprendizaje, ya no estaría el sujeto, sino las actividades, que deberían impulsar un papel activo del sujeto en diversidad de experiencias que incidan en su constitución, es decir, en su agencialidad (Gutierrez y Rogoff, 2003). De esta manera, el aprendizaje se concibe como una práctica situada, que propicia el desarrollo de la educabilidad (Baquero, 2006) de los sujetos, pues se trata de recuperar la confianza en las posibilidades de aprendizaje de los jóvenes, y generar experiencias educativas genuinas y potentes. Esto supone un enfoque centrado en el sujeto en situación. En dicho enfoque, el aprendizaje no se concibe como la capacidad de aprender que porta un sujeto, sino que se explica en función de los vínculos intersubjetivos en las situaciones en las que participa: el aprendizaje resulta de los procesos de interacción entre los sujetos y la institución. En ese sentido, el aprendizaje puede definirse como los cambios que se producen en las formas como comprenden y participan los sujetos en una actividad conjunta (Baquero, 2006). Además de ser distribuido entre los sujetos, es heterogéneo, múltiple y diverso en cuanto a producción de significados y conocimientos. Justamente, por ello, el docente debe asumirse como un sujeto capaz de elaborar una propuesta de enseñanza que articule las lógicas disciplinares, las posibilidades de apropiación por parte de los sujetos y el contexto. En definitiva, el docente universitario debería intentar construir puentes entre su propia comprensión como experto de una disciplina y la comprensión inicial del estudiante. Asimismo, debería generar propuestas que involucren problemas y prácticas propios de su campo profesional.

Otra cuestión medular en este marco teórico es la labor tutorial, que está estrechamente vinculada con el proceso de aprendizaje de los estudiantes.

12 I En ese marco, constituye un aporte teórico el realizado por Clérici y Amieva 
(2013), quienes identifican diferentes perspectivas de los docentes ingenieros con respecto a la tutoría. Una primera perspectiva considera a la tutoría como actividad meramente formal, debido a que los tutores realizan una tarea burocrática, con una lógica administrativa, y aguardan la demanda de orientación que pudiera surgir por parte de los estudiantes. Desde otro punto de vista, la tutoría es concebida como una actividad asistencialista, y con la mirada puesta en el déficit de los estudiantes y en problemáticas ya instaladas. De esta manera, la tutoría se convierte en un espacio marginal con respecto al currículum y a la formación de grado. Entendiendo un nuevo rol de la tutoría, otra perspectiva ubica su quehacer desde una construcción situada, que, en contraposición con la docencia tradicional, contempla las particularidades de los estudiantes, las necesidades de la institución y los intereses de los tutores. Finalmente, desde la última perspectiva, la tutoría es entendida como una función docente. Las autoras afirman que esta mirada implica una revisión del rol docente, pues la función de orientación es inherente a este quehacer y no debería ser asignada a un grupo específico. En esa línea, el docente debería orientar el aprendizaje de los estudiantes en función del marco de cada materia.

Al respecto, un aporte disciplinar de Leticia Moreno (2010) consiste en su aproximación a la tutoría en articulación con la docencia y su identificación de tres supuestos teóricos. El primero consiste en la tesis de la disociación de la tutoría como ayuda a la enseñanza, ofrecida en un horario específico y orientada al programa de las materias. El segundo corresponde a la tesis de la complementariedad, que implica estrategias de orientación en tutorías grupales e individuales. En este caso, la tutoría es un complemento de la formación (orientación personal, profesional, académica). El tercero es entendido como la tesis de la integración, en la que ambas funciones se integran.

Un aporte teórico clave para la comprensión de las prácticas tutoriales lo constituyen las cuatro configuraciones del rol del tutor que identifica Capelari $(2009,2016)$ en las universidades argentinas. La autora analiza de manera multidimensional los programas de tutoría, y afirma que conocer los motivos y los problemas a los que responde la existencia del tutor es clave, porque de ellos se desprende el direccionamiento que toma el espacio que configura su rol. Entre los principales motivos, identifica tres: a) la solución de problemas situados en una población: los estudiantes; b) la respuesta personalizada a las problemáticas de enseñanza y de aprendizaje de los estudiantes a través de lo relacional y/o lo intersubjetivo; y c) la optimización de los aprendizajes en articulación y/o los cambios en el sistema de actividad institucional.

Estos motivos orientan las diferentes configuraciones del rol del tutor:

a) El tutor como parte de un dispositivo institucional de tipo remedial para solucionar dificultades situadas en estudiantes: el objetivo de sus intervenciones es impactar sobre los índices de abandono y la reprobación de materias, detectar estudiantes en situación crítica, entre otros. Estos dispositivos tutoriales están poco articulados con las demás actividades académicas. 
b) El tutor como orientador que brinda respuestas personalizadas a distintas necesidades y problemas de los estudiantes: se trata de una actividad tutorial proactiva, centrada en potenciar al estudiante. Por eso, el aprendizaje es uno de los aspectos en los que se interviene, pero no el único.

c) El tutor como orientador/promotor de aprendizajes académicos: el tutor cumple un rol proactivo, pues responde de manera personalizada a las problemáticas de aprendizaje planteadas por los estudiantes para potenciar nuevos conocimientos, habilidades o actitudes. Dentro de esta configuración, la labor del tutor está inserta en la institución, pero su lugar en la estructura organizativa es variable.

d) El tutor como una forma especial de ser docente: en este caso, enseña a través de un formato pedagógico innovador que le permite ocupar un lugar clave en los aprendizajes, y en el marco de los planeamientos curriculares y de concepciones sobre el currículum, la didáctica, el aprendizaje y la enseñanza. Este tutor está inserto en la institución y ocupa un lugar central. En palabras de Moreno (2010), se trataría de un docente-tutor que acompaña al estudiante en su desarrollo integral. Esta figura se concibe "como un mediador capaz de crear condiciones que permitan que los saberes tengan sentido para los estudiantes, lo cual exige un amplio conocimiento de las dimensiones epistemológicas, sociales e históricas de su asignatura" (Moreno, 2010, p. 99).

En línea con esta autora, Capelari (2009) entiende que es necesario analizar críticamente los motivos y los objetivos de los programas de tutoría que se implementan en las instituciones de educación superior. Asimismo, señala que es preciso cuestionarse los efectos de estos procesos en la constitución de los sujetos y su posibilidad de aprender, así como revisar los sentidos atribuidos a las causas del "fracaso" y "éxito" de los alumnos. Estos aportes de la autora permiten reflexionar sobre las maneras en que los tutores docentes conciben la noción de fracaso educativo, pues, a partir de esas representaciones, se manifiesta su accionar desde su rol como tutor. Los sentidos que adquiere el fracaso educativo son claves dentro de la labor tutorial, debido a que el surgimiento de los sistemas de tutoría y las intervenciones que realizan suelen estar orientados hacia la necesidad de revertir el fracaso educativo en el nivel superior.

En suma, la literatura revisada permite acercarse desde diferentes aristas a los múltiples aspectos que se imbrican en la labor tutorial, en el desempeño de los docentes universitarios y tutores, y en la construcción del conocimiento que realizan los estudiantes en un intercambio permanente con docentes y tutores. En dicho intercambio, comparten y negocian significados para apropiarse de saberes y fortalecer las competencias comunicativas, que les permitirán desenvolverse en su quehacer académico y profesional. 


\section{Metodología}

Se ha optado por realizar un estudio cualitativo de carácter exploratorio. El diseño ha sido de carácter empírico no experimental a fin de captar, a través de distintos instrumentos, el punto de vista de los agentes en relación con el enfoque didáctico que fundamenta el Programa de Tutoría, según las particularidades del contexto en que este se desarrolla.

El problema, los objetivos y el enfoque teórico desarrollado constituyen el marco del cual emergen las dimensiones y las subdimensiones que se tuvieron en cuenta para construir los instrumentos de recolección de datos y realizar el análisis. Las dimensiones y las subdimensiones se conciben como distintas aristas de una misma realidad: están estrechamente relacionadas y su interrelación hace posible la construcción de los múltiples sentidos que adquiere el Programa de Tutorías, desde la perspectiva de los tutores y de quien lo coordina. Sin embargo, en este trabajo, se establecen distinciones entre las dimensiones, a fin de indagar en detalle y con mayor profundidad los significados que se le atribuye a cada una.

En la siguiente tabla, se pueden observar las dimensiones y las subdimensiones de análisis construidas para este trabajo.

Tabla 1. Dimensiones y subdimensiones de análisis

\begin{tabular}{l} 
Dimensiones \\
\hline Caracterización del enfoque didáctico del \\
Programa de Tutorías Académicas en Com- \\
petencias de Lectura y Escritura
\end{tabular}

Significados atribuidos a la relación entre los saberes a enseñar y la escritura académica Construcción del vínculo didáctico entre docentes ingenieros y tutores en competencias de lectura y escritura

Fortalezas y debilidades del enfoque didáctico del Programa de Tutorías

Cambios impulsados por la implementación del programa

Transformaciones propuestas

\section{Subdimensiones}

Problemas a los que busca responder el enfoque didáctico del Programa de Tutorías Académicas en Competencias de Lectura y Escritura

Principales objetivos del enfoque didáctico del Programa de Tutorías Académicas en Competencias de Lectura y Escritura

Responsabilidades que asume cada uno de los integrantes de la pareja pedagógica

Cambios en los aprendizajes de los estudiantes

Cambios en las prácticas de enseñanza de los docentes

Cambios en las prácticas de escritura de los estudiantes

Fuente: Urús, 2016. 
Las herramientas utilizadas para la generación de datos fueron un cuestionario individual y el análisis documental. Por un lado, se eligió el cuestionario, debido a que otorga la posibilidad de realizar preguntas estructuradas y/o semiestructuradas para indagar las perspectivas de los sujetos sobre las distintas categorías de análisis definidas. Por otro lado, se realizó un análisis documental que incluyó los principales documentos institucionales asociados al programa en el que participan los sujetos. Para su selección, se tomó en cuenta que fueron escritos por quienes diseñaron o llevaron adelante la implementación del programa. Los documentos seleccionados fueron Programa Institucional de Tutorías. Un modelo Integral (2008), cuyas autoras son la doctora Liliana Laco y la licenciada Lorena Guiggiani, y Una experiencia en alfabetización académica: evolución, análisis y resultados (2012), de la doctora Liliana Laco y la profesora Mónica Ávila.

Los datos producidos se organizan en matrices de contenido según su relación con las dimensiones y las subdimensiones de análisis. En el análisis, se prioriza la técnica de análisis del discurso para considerar los significados atribuidos al programa en sus distintas dimensiones.

Los sujetos que participaron en este estudio incluyeron a cuatro docentes y a la coordinadora del programa. La información del perfil de cada uno de los sujetos participantes puede observarse en la siguiente tabla, que muestra los códigos de registro de la participación de los docentes tutores y de la coordinadora en el cuestionario individual.

Tabla 2. Códigos de registro de los sujetos participantes

\begin{tabular}{|c|c|c|c|c|c|c|}
\hline $\begin{array}{l}\text { Código de } \\
\text { registro en } \\
\text { cuestionarios }\end{array}$ & Género & Edad & Profesión & Asignatura & $\begin{array}{c}\text { Rol que } \\
\text { desempeña }\end{array}$ & $\begin{array}{c}\text { Antigüe- } \\
\text { dad en el } \\
\text { cargo }\end{array}$ \\
\hline Docente 1 & Masculino & 50 & $\begin{array}{l}\text { Ingeniero } \\
\text { electricista }\end{array}$ & $\begin{array}{l}\text { Integración } \\
\text { Eléctrica II }\end{array}$ & $\begin{array}{l}\text { Profesor } \\
\text { Adjunto }\end{array}$ & Dos años \\
\hline Docente 2 & Masculino & 30 & $\begin{array}{l}\text { Ingeniero } \\
\text { electricista }\end{array}$ & $\begin{array}{l}\text { Integración } \\
\text { Eléctrica I }\end{array}$ & $\begin{array}{c}\text { Jefe de } \\
\text { Trabajos } \\
\text { Prácticos }\end{array}$ & $\begin{array}{l}\text { Cuatro } \\
\text { años }\end{array}$ \\
\hline Docente 3 & Femenino & 33 & $\begin{array}{l}\text { Ingeniera } \\
\text { mecánica }\end{array}$ & $\begin{array}{l}\text { Ingeniería } \\
\text { Mecánica II }\end{array}$ & $\begin{array}{c}\text { Profesora } \\
\text { Adjunta }\end{array}$ & Un año \\
\hline Docente 4 & Masculino & 55 & & $\begin{array}{l}\text { Integración } \\
\text { Eléctrica I }\end{array}$ & Ayudante & $\begin{array}{l}\text { Veintiún } \\
\text { años }\end{array}$ \\
\hline Coordinadora & Femenino & 61 & $\begin{array}{c}\text { Profesora } \\
\text { de Lengua } \\
\text { y Literatura }\end{array}$ & & $\begin{array}{c}\text { Coordina- } \\
\text { dora del } \\
\text { Programa } \\
\text { de Tutoría } \\
\text { en Compe- } \\
\text { tencias de } \\
\text { Lectura y } \\
\text { Escritura }\end{array}$ & $\begin{array}{c}\text { Nueve } \\
\text { años }\end{array}$ \\
\hline
\end{tabular}




\section{Resultados}

Los datos producidos exhiben tensiones, continuidades y rupturas en las configuraciones didácticas que los actores involucrados - docentes y tutor-ponen en juego en el quehacer de aula. Dichas tensiones manifiestan aspectos epistémicos e identitarios en relación con los sentidos que adquiere la formación de un profesional que será ingeniero, y subyacen en las concepciones desde las que se construyen las prácticas docentes. En este apartado, se presenta una interpretación de los datos, a partir de la teoría expuesta en el marco teórico y considerando cada una de las dimensiones propuestas.

\section{Caracterización del enfoque didáctico del programa}

La información muestra que se establece una estrecha relación entre los problemas a los que el programa busca responder y los objetivos que plantea. Según los testimonios, tanto los motivos como los objetivos se asocian a solucionar las carencias de los estudiantes. Como caracteriza Capelari (2009), se encuentra que los motivos fundamentales que orientan los propósitos de la existencia de la tutoría responden a solucionar problemas situados en una población: los estudiantes. Por ejemplo, los docentes consideran que se trata de "atacar, intentar contrarrestar" (Docente 4) distintas dificultades, como "reducir errores de ortografía, desarrollar el hábito de la lectura comprensiva y fomentar la escritura argumentativa" (Docente 1). Para uno de los cuatro docentes, el propósito es "mejorar las capacidades discursivas de los alumnos" (Docente 2) para "compensar el bajo nivel de expresión oral y escrita que traen los alumnos" (Docente 2). Además, en uno de los testimonios, se incluye dentro de los motivos y de los objetivos a los docentes, percibidos desde una mirada deficitaria. Este pensamiento se manifiesta de la siguiente manera:

Este problema no es solo de los alumnos, ya que los docentes han tenido una formación similar, por lo cual, muchas veces, se ven afectados por la misma problemática. Esto se refleja en sus clases y en los apuntes que preparan, así como también en sus presentaciones de diapositivas y en su manera de explicar conceptos nuevos que los alumnos no comprenden. (Docente 3 )

A partir de los relatos de los sujetos, se percibe que el condicionante que predomina en la institucionalización de este programa es la inclusión de la tutoría en los planes de mejora de las carreras para la solución de problemas de ingreso, permanencia y graduación de los estudiantes.

En una de las publicaciones del proyecto analizado (Laco y Guiggiani, 2008), los objetivos que se enuncian se relacionan con el motivo que los docentes exponen. Es decir, los objetivos dan cuenta de los motivos institucionales. En un documento generado cuatro años después (Laco y Ávila, 2012), se 
encuentran cambios en los objetivos. Se percibe la tutoría como una forma de intervención con una estrategia específica - el trabajo interdisciplinario-e, incluso, articulada a diferentes componentes del sistema de actividad institucional (Capelari, 2016), como el currículum, la formación docente y la construcción de estrategias interdisciplinarias.

En algunos casos, se ha hallado que, en la percepción del docente que acompaña al tutor, prevalece la mirada puesta en una problemática considerada ya instalada: la carencia de los estudiantes y, por ende, la tutoría como una actividad asistencialista, centrada en el déficit (Clérici y Amieva, 2013). En otras palabras, algunos docentes parecen considerar a los estudiantes desde las teorías del déficit y de la diferencia. Desde estas aproximaciones, entienden que la tutoría debe cumplir el objetivo de "remediar" la situación inicial con que ingresan los estudiantes. Es posible que esta perspectiva se haya consolidado a través de las transformaciones de sentido de la educación pública, que se incorporaron desde las políticas educativas en la década del noventa. El Plan Social Educativo promovió políticas compensatorias focalizadas en determinadas poblaciones, definidas desde el parámetro del déficit. Desde esta perspectiva, por un lado, se sitúa a los sujetos en una posición de subordinación, de asistido y privado de la palabra. Por otro, se niega el carácter social y complejo del problema, pues se tiende a atribuir a los sujetos la causa del mismo.

Sin embargo, otros testimonios y documentos consultados demuestran que, en simultaneidad con estas miradas, conviven perspectivas diferentes de otros docentes del programa. Estos docentes entienden que la tutoría debería integrarse a la enseñanza de grado, de manera tal que acción tutorial y acción docente formen un nuevo esquema pedagógico de acción y apoyo al estudiante (Clérici y Amieva, 2013), puesto que parten de la posibilidad de seguir desarrollando competencias comunicativas tanto en los estudiantes como en los docentes. En definitiva, desde la mirada de los actores involucrados, el enfoque didáctico de este programa de tutorías se caracteriza por perspectivas diversas y en tensión entre sí. La convivencia de estas concepciones expone la complejidad de la tarea entre profesionales de distintas áreas y el marco desde el que se construyen los cambios en el quehacer en el aula.

\section{Significados atribuidos a la relación entre los saberes a enseñar y la escritura académica}

Los datos producidos muestran que los docentes se van acercando a la noción de alfabetización académica (Carlino, 2005, 2013) cuando reconocen que las prácticas de escritura propuestas en las clases se realizan a partir del contenido disciplinar que desarrollan. Sin embargo, no se identifica la dimensión epistémica de la escritura, en términos del análisis que se realiza, y se observa que establecen una relación con el saber -en este caso, de las prácticas de escritura- a través de la figura del aprender denominado por Charlot (2008) como "objetivación-denominación". Desde esta relación, aprender implica que 
un sujeto consciente pase de identificar un saber virtual a su apropiación real. El lenguaje es el instrumento que da cuenta de cómo se realiza ese pasaje y la apropiación de ese objeto. Esta postura se refleja cuando los docentes manifiestan que incorporan prácticas de escritura - por ejemplo, los exámenes parciales - para que los estudiantes puedan mostrar lo que han aprendido y los docentes puedan evaluarlo. En otras palabras, los docentes establecen una relación de objetivación-denominación con el saber sobre las prácticas de escritura, en la medida que la escritura aparece como un conocimiento práctico para resolver la situación de examen parcial, en la cual los estudiantes deben dar cuenta de la posesión del saber-objeto específico de la cátedra. La escritura, en ese sentido, se concibe como una herramienta que permite observar el resultado de lo aprendido, a través de lo que los estudiantes comunican.

Dos testimonios manifiestan que, a partir de la inclusión de la tutoría, los docentes implementan actividades prácticas como la escritura de informes y de argumentaciones (orales y escritas), que son actividades que responden a los requerimientos del ámbito profesional. Por ejemplo, un docente explica:

Se les solicita a los estudiantes que presenten una determinada propuesta. A esta propuesta los docentes presentan objeciones adrede (como suele suceder habitualmente en el ámbito profesional) para que argumenten una respuesta justificada y así, de esta manera, ejercitar el hábito de la argumentación que todo profesional debe dominar. (Docente 1)

En estas propuestas didácticas, se percibe que los docentes comienzan a otorgarles un lugar preponderante a las actividades que les ofrecen a los estudiantes. De este modo, se intentarían desarrollar experiencias genuinas y potentes, y se evitaría caer en la utilización de métodos generales ajenos a la especificidad de los contenidos disciplinares y de la formación profesional para propiciar la buena enseñanza, en términos de Litwin (1996).

En uno de los documentos analizados (Laco y Ávila, 2012), se indica que los docentes suelen evaluar aspectos superficiales del proceso de escritura y que no suelen considerar erróneos aquellos textos que están mal expresados. Ello podría suponer una desvalorización de la competencia cognitiva que implica el auténtico proceso de escritura e incide en las prácticas de enseñanza. Es decir, la configuración didáctica que subyace a este accionar no favorecería procesos significativos de construcción de conocimiento (Litwin, 1997), en los que se amalgamen las lógicas disciplinares, las posibilidades de apropiación de los sujetos y el contexto de aprendizaje.

Estas tensiones - que conviven en el aula- muestran que las prácticas de escritura no surgen "naturalmente" como una necesidad inherente a la disciplina. Posiblemente, esto sea aprendido y valorado por los estudiantes de la misma forma en que lo transmiten sus docentes. 


\section{Construcción del vínculo didáctico entre docentes ingenieros y tutores en competencias de lectura y escritura}

Los significados analizados en esta dimensión emergen de las actividades que comparten docentes y tutores, y muestran la coexistencia de diferentes posturas, muchas veces, contradictorias. Por un lado, cuando los actores sostienen que se vinculan a través de reuniones - en las que surgen distintas reflexiones sobre múltiples aspectos de su quehacer didáctico-, la tutoría aparece integrada a la enseñanza de grado, puesto que la acción tutorial y la acción docente comenzarían a formar un nuevo esquema pedagógico (Clérici y Amieva, 2013; Moreno, 2010). En los primeros pasos de esta integración, los docentes universitarios estarían construyendo un nuevo enfoque pedagógico que contemplaría las siguientes etapas como modalidad de trabajo: estudio, tutoría, trabajo conjunto, evaluación y satisfacción mutua (Moreno, 2010). Asimismo, se podría afirmar que el motivo que orienta esta actividad, y se traduce a través de este accionar, se relaciona con una configuración tutorial asociada a optimizar los aprendizajes mediante la articulación y/o los cambios en otros componentes del dispositivo institucional (Capelari, 2016).

Sin embargo, al analizar cómo se concibe el rol de cada uno de los integrantes de esta pareja pedagógica, se observa que conviven simultáneamente diversos motivos de la actividad (Capelari, 2009, 2016). Por ejemplo, cuando los docentes distinguen de manera tajante las actividades que cada uno debe realizar y se responsabiliza al tutor de las tareas que atañen a la lectoescritura, prevalece una perspectiva de la tutoría disociada de la enseñanza y asociada a un dispositivo de tipo remedial para solucionar dificultades situadas en los estudiantes. Es decir, el rol ejercido por el tutor correspondería a la configuración que identifica Capelari (2009) vinculada con la solución de problemas situados en una población: los estudiantes. Además, se alinea con uno de los motivos que, mayoritariamente, orientan la implementación de los programas de tutoría.

También, se ha encontrado otra contradicción que surge en los documentos analizados. En ellos, se destaca que el tutor realiza su labor desde dentro de la cátedra "acompañando" a los docentes. Sin embargo, ese "estar dentro" -que distingue la implementación de este programa- no significa que los docentes se apropien de la perspectiva de trabajo del tutor, tal como se evidencia en dos testimonios. En uno de ellos, el docente expresa: "El rol de la tutora se enmarca en la intervención en algunas clases prácticas programadas con la asistencia puntual en las competencias de lectura y escritura" (Docente 1). En otras palabras, el rol del tutor - además de adquirir un carácter remedialresponde a problemáticas de aprendizaje planteadas por los estudiantes y sus intervenciones se circunscriben a cuestiones relacionadas con los aprendizajes académicos de los jóvenes. Entonces, el "acompañamiento" es para los estudiantes, no para los docentes. Como consecuencia, el motivo del programa que subyace a este accionar entra en tensión con lo expresado en los documentos. 
Se sigue manifestando la creencia de que las tutorías tienden a responder de manera personalizada a los problemas de aprendizaje de los estudiantes (Capelari, 2016), que se convierten en objetos-sujetos de la intervención tutorial. En definitiva, en algunos casos, pareciera que el vínculo que se establece dentro de la pareja pedagógica, por un lado, es poco permeable a que los docentes se apropien de cambios en su rol y, por otro lado, reduce el quehacer del tutor a resolver problemáticas identificadas en un grupo de sujetos de aprendizaje. De este modo, parece difícil dirigir una mirada en la que se torna más intrincado que docente y tutor puedan identificar la complejidad de las prácticas áulicas, y propongan cambios en sus roles y en los objetivos de la intervención docente.

No obstante, otros testimonios dan cuenta de que la construcción del vínculo entre ambos ha logrado un nivel de imbricación que permite afirmar que los docentes han comenzado a concebir el rol de tutor como una forma especial de ser docente (Capelari, 2009). Esto responde a que, en la tarea colaborativa y conjunta que realizan, los docentes se van apropiando lentamente del enfoque didáctico que proponen los tutores, y lo van incorporando en sus concepciones de didáctica, currículum, aprendizaje y enseñanza. Al respecto, un docente expresa que ha construido un "vínculo de trabajo colaborativo que permite una completa implementación de las competencias que se desean abarcar" (Docente 3 ).

En suma, la manera de construir vínculos entre docentes y tutores es diversa, en función de las responsabilidades atribuidas a cada rol, y de los significados y motivos asociados al sentido del programa. Estos dos últimos aspectos están emparentados a las concepciones que tiene cada actor con respecto a las implicancias del proceso de enseñanza en una carrera de grado de Ingeniería.

\section{Fortalezas y debilidades del enfoque didáctico del Programa de Tutorías Académicas en Competencias de Lectura y Escritura}

Las respuestas de los docentes reflejan algunas de las tensiones que ya fueron mencionadas. En cuanto a las fortalezas, se considera positivo el aporte de "modelos y estructuras textuales" (Docente 4), así como el "apoyo" (Docente 2) y el "respaldo" (Docente 4) de la tutoría ante el déficit de los estudiantes. Esta perspectiva, desde los posicionamientos teóricos a los que se adhiere en el trabajo, constituye una modalidad que invisibiliza condicionantes institucionales y de enseñanza que son fundamentales en el logro de los aprendizajes. Al ubicar a la tutoría en una configuración de tipo remedial, según Capelari (2009), el tutor solo interactúa con los estudiantes "en riesgo" - desde las competencias comunicativas-, sin articular su intervención con otros aspectos de la actividad académica del sujeto ni con la tarea docente.

Entre las fortalezas, también, se menciona la posibilidad que tienen los estudiantes de articular las prácticas de escritura con la actividad profesional, en tanto las intervenciones tutoriales se realizan respetando el contexto académico propuesto por los docentes de cátedra. Este tipo de relación ha sido 
cuestionado en función de los planteamientos de Bombini (2009), quien sostiene que la escritura que se realiza en el ámbito académico nunca puede recrear las prácticas que realmente ocurren en el ámbito laboral. No obstante, otros autores, como Zavala (2009), consideran que, a medida que los estudiantes van observando e interactuando con los modos de hablar, actuar, valorar, pensar, sentir de la comunidad a la que desean pertenecer, esas formas de comunicarse se van volviendo naturales en ellos. En la identificación de esta fortaleza, se observa que las propuestas de prácticas de escritura relacionadas con el ámbito laboral procuran acercar a los estudiantes al modo de comportarse y comunicarse que caracteriza a los ingenieros. Según la teoría de la acción comunicativa, en esas actividades, se comparten y se construyen sentidos, en este caso, en torno a la profesión de los ingenieros (Litwin, 1996).

Por último, otras fortalezas que se mencionan están relacionadas con la posibilidad de darle continuidad a la tarea que se realiza en el Seminario de Ingreso y se valora positivamente el cambio didáctico que implica "la posibilidad de realizar una planificación conjunta" (Docente 3) entre docentes y tutora. Esto significa que, por un lado, tanto estudiantes como docentes se van apropiando de la legitimidad que tiene la enseñanza y el aprendizaje de la escritura académica en el ámbito universitario (Carlino, 2005, 2013), pues la continuidad del trabajo consolida esta postura. Por otro lado, se va complejizando la configuración didáctica de las cátedras en aras de habilitar espacios en los que la enseñanza de competencias comunicativas sea comprensiva (Litwin, 1997).

Con respecto a las debilidades y los problemas que se identifican en la implementación del enfoque didáctico, cabe evaluar si los docentes se han apropiado de las posibilidades epistémicas que son inherentes a las prácticas de escritura. En este sentido, cuando se afirma que la cátedra no tiene demasiado tiempo de aula para "intercalar, dentro del desarrollo de lo específico, las actividades de lecto-escritura" (Docente 4), se percibe que estas actividades están disociadas aún para algunos docentes, quienes consideran las prácticas de escritura, por un lado, y el desarrollo de los contenidos disciplinares, por otro. En otras palabras, a través de este tipo de afirmaciones, se muestra que aún no se concibe la posibilidad de enseñar y aprender el contenido disciplinar utilizando las prácticas de escritura como estrategia didáctica.

Otra debilidad señalada es la resistencia de los estudiantes ante las actividades de escritura. Esta resistencia también es manifestada por los docentes cuando, por ejemplo, en las correcciones de textos o devoluciones, no consideran importantes las cuestiones de elaboración textual para definir la nota de un examen parcial. Es decir, si bien los docentes no desvalorizan las prácticas de escritura de forma explícita, cuando no las consideran en sus devoluciones o no les otorgan una calificación dentro de las instancias de evaluación, implícitamente se desplaza el desarrollo de las competencias comunicativas con respecto al contenido disciplinar. Además, cuando se considera como una debilidad que el tutor no pueda estar más tiempo en sus cátedras, se está reforzando una configuración remedial de dicho rol, que estaría centrado en responsabilizarse 
de las dificultades comunicativas de los estudiantes y solucionarlas para que puedan avanzar. Ello manifiesta que los docentes no conciben la posibilidad de incluir el enfoque que propone el Programa de Tutoría en su configuración didáctica. En este enfoque, la escritura deja de ser una actividad aislada y se la entiende como una estrategia de enseñanza. Esta última cuestión también es planteada por la coordinadora del programa, quien sostiene que el estilo de trabajo de algunas cátedras ha dificultado la incorporación de cambios en el quehacer en el aula.

\section{Cambios impulsados por la implementación del Programa de Tutorías Aca- démicas en Competencias de lectura y Escritura}

En los cambios que los distintos actores consideran necesarios, se observan coincidencias con algunas de las tensiones que se presentan en el análisis de otras dimensiones. En cuanto a los cambios en los aprendizajes de los estudiantes, aparece nuevamente el predominio de la figura del aprendizaje objetivación-denominación (Charlot, 2008), pues se valora la forma como el estudiante transmite la posesión del saber-objeto específico de la cátedra, a través de la escritura. En este marco, la escritura es considerada como una herramienta "transparente", que permitiría observar lo que el estudiante ha aprendido para ser evaluado. Por ejemplo, cuando se afirma que "hay mejoras en el decir de lo aprendido" (Docente 4), se percibe que el docente evalúa el aprendizaje del contenido disciplinar, a partir de lo que el estudiante escribe. Un docente destaca que los estudiantes comprenden que pueden utilizar estas competencias comunicativas en el ámbito laboral y que van construyendo "una perspectiva holística de cómo debe desempeñarse un ingeniero" (Docente 3). También, se valora positivamente que los estudiantes puedan "aplicar tipos de texto" (Docente 2) "en las formas de presentación de informes" (Docente 1). Cabe mencionar la crítica y la advertencia que plantea Bombini (2009) con respecto a la reducción que se realiza de las prácticas de escritura universitarias cuando se privilegian las tipologías textuales. Bombini (2009) sostiene que "se trata de una ilusión de intervención remedial, a la manera de un antídoto científico sobre las «malas prácticas» o las «bajas competencias»" (p. 434). En otras palabras, la valoración que hacen los docentes sobre el manejo de tipologías textuales se relaciona con el tipo de intervención tutorial, en el cual se hace hincapié en tipos de texto, como el informe o el abstract, tal como señala la coordinadora. Entonces, siguiendo el planteamiento de Bombini (2009), se podría afirmar que algunas de las estrategias utilizadas por el enfoque didáctico del programa pueden reforzar la construcción de una representación de tipo remedial de la propuesta, que presenta y obstaculiza el desarrollo de aprendizajes genuinos y con sentido para los sujetos.

En cuanto a los cambios en las prácticas de enseñanza de los docentes, se observa que cada uno de ellos va complejizando la construcción de su propia configuración didáctica, en términos de Litwin (1997). Los docentes afirman 
que se han apropiado de "un discurso nuevo del valor pedagógico del uso de las herramientas de lectoescritura" (Docente 4), han incorporado "herramientas de competencias de escritura" (Docente 1), han logrado ser "más precisos en la elaboración de consignas" (Docente 2) y han añadido "otros aspectos en el momento de realizar la corrección" (Docente 3). A través de estas afirmaciones, dan cuenta de que se van volviendo más expertos en el despliegue de estrategias y actividades genuinas que favorecen procesos de construcción del conocimiento, en la medida que facilitan que los estudiantes comiencen a formar parte del mundo simbólico que se construye en el funcionamiento discursivo con sus pares (Baquero, 2001; Mercer, 1997; Rogoff, 1993; Zavala, 2009). Asimismo, la coordinadora destaca que la inclusión de prácticas de escritura en el aula ha producido "impactos de tipo didáctico": los docentes no solo han revisado "cuestiones vinculadas con la escritura, sino aspectos que tienen que ver con la gradualidad de dificultades" que les presentan a los estudiantes.

Por último, con respecto a los cambios en las prácticas de escritura de los estudiantes, los docentes identifican que estos aprenden a manejar distintos aspectos del proceso de escritura (coherencia, cohesión, tipología textual). Además, identifican como relevante el aspecto del formato textual y, en esta subdimensión, revelan que los jóvenes también se apropian del manejo de niveles textuales más complejos, como la coherencia y la cohesión. Entonces, se observa cómo los docentes van asumiendo el enfoque didáctico propuesto por la noción de escritura académica (Carlino, 2005, 2013), en tanto distinguen aspectos lingüísticos en las producciones escritas y los valoran positivamente. Finalmente, es importante la anotación de la coordinadora del programa, quien pone de relieve cómo la demanda de prácticas de escritura que los docentes plantean ha logrado "progresos sostenidos en las producciones escritas de los alumnos". Se podría afirmar, entonces, que esa continuidad favorece la construcción de algunos espacios caracterizados por la buena enseñanza (Fenstmarcher, 1989) y la enseñanza comprensiva (Litwin, 1997).

\section{Transformaciones propuestas}

En las transformaciones sugeridas por los docentes, aparece la necesidad de realizar capacitaciones periódicas para docentes y estudiantes, sumar recursos humanos para atender los requerimientos de los estudiantes de forma personalizada y expandir el programa a otras cátedras. Estas propuestas sugieren que, a través del trabajo de estos años, se ha internalizado la importancia de generar espacios para el ejercicio de la reflexión entre pares. Aparece la necesidad de que se reúnan los docentes involucrados para dialogar sobre las "metas, objetivos y estrategias" (Docente 4) propuestas por el programa, a fin de que los docentes "las internalicen y se motiven" (Docente 4) para aplicarlas. También, surge la posibilidad de que, en esas reuniones, los docentes puedan "practicar la producción de textos y la corrección de producciones ajenas" (Docente 3). Sin embargo, cuando se propone "capacitar a los alumnos" (Docente 
1) y "aumentar los recursos humanos para hacer un seguimiento del alumno personalizado" (Docente 2), se refleja nuevamente la dificultad que implicaría la posibilidad de que los docentes incorporen a su rol la enseñanza de las prácticas de escritura inherentes al desarrollo del contenido disciplinar. Por ello, pareciera que la mirada hacia el estudiante se enfoca en sus carencias desde la teoría del déficit. Por último, al sugerirse expandir los recursos a "otras materias compatibles con la tutoría" (Docente 2), aparece nuevamente la tensión entre diferentes maneras de concebir la construcción del conocimiento, y el lugar que tienen las competencias comunicativas y el rol del tutor en esa construcción, puesto que pareciera que habría materias que no serían compatibles con las actividades de lectura y escritura.

\section{Reflexiones finales y contribuciones}

La interpretación de los datos producidos a la luz de la teoría muestra la compleja trama de continuidades, diferencias, escisiones y tensiones que conviven en el interior de algunas de las cátedras universitarias en las que se desarrolla el Programa de Tutorías en Competencias de Lectura y Escritura.

Las principales contradicciones y resistencias que se manifiestan reflejan concepciones - sobre el aprendizaje, la enseñanza, el rol docente, los contenidos disciplinares en el ámbito universitario, el estudiante, la tutoría, las prácticas de escritura - fuertemente arraigadas a la tradición educativa enciclopédica, producto de construcciones socioculturales e históricas. Los conflictos surgen cuando los docentes, con esos marcos interpretativos, valoran el enfoque que propone la tutoría y toman decisiones didácticas para enseñar desde nuevos enfoques epistemológicos, psicoeducativos y didácticos. Las incongruencias, que se observan en los testimonios, hacen explícitos estos aspectos epistémicos y están vinculadas con las tensiones que surgen entre las representaciones sobre la enseñanza, el aprendizaje, la formación profesional del ingeniero y la subjetividad de cada uno de los docentes que participan del programa.

Los docentes oscilan entre dos posturas: les otorgan importancia a las prácticas de lectura y escritura, pero parecen no concebir la posibilidad de apropiarse de ellas, en tanto estrategias de enseñanza. Se percibe aún una escasa valoración al momento de calificar los trabajos de los estudiantes y consideran que es responsabilidad de la tutoría resolver las dificultades de lectura y escritura que estos pudieran tener.

Si bien prevalece en varios actores una mirada remedial sobre la función de este programa de tutoría, no se desestima el enfoque didáctico propuesto. Incluso, en algunos casos, se vislumbra una lenta transición hacia configuraciones didácticas más complejas, en las que se comienza a concebir que las competencias comunicativas son inherentes a los contenidos abordados en las cátedras universitarias.

En relación con las contribuciones de este trabajo, y teniendo en cuenta los propósitos definidos en su inicio, se ha logrado realizar un primer estudio 
exploratorio e integral sobre el programa, en el que se ha incluido a algunos de los actores involucrados. Esto constituye un aporte importante de este trabajo académico, pues se trata de presentar una primera evaluación al enfoque didáctico de un programa de tutoría que se está implementando desde hace nueve años en la Universidad Tecnológica Nacional, Facultad Regional General Pacheco.

En líneas de trabajo a futuro, se considera importante complejizar este primer acercamiento al objeto de estudio y obtener una mirada holística del programa. Una posibilidad valiosa sería avanzar con la realización de un estudio a profundidad y explicativo, que permita ampliar la cantidad de docentes participantes y utilizar otros instrumentos de recolección de datos que posibiliten indagar en mayor profundidad y complejidad los aspectos esbozados en el presente trabajo.

Los resultados alcanzados sugieren, también, la posibilidad de generar líneas propositivas de mejora para el programa. Entre las mismas, podrían señalarse la planificación de jornadas de capacitación para los docentes con el fin de habilitar espacios de reflexión sobre distintos aspectos de la escritura académica dentro de la didáctica universitaria.

De acuerdo con los resultados del análisis realizado, la organización de las temáticas a tratar podría considerar una secuencia, de manera tal que los docentes puedan vincular sus prácticas con la teoría para construir nuevos significados y retornar al aula con nuevas actividades. El recorrido que se propone podría iniciarse a partir de la narración de experiencias en el aula realizadas por los docentes sobre una temática puntual. Luego, estas pueden ser contrastadas con posturas teóricas actuales. A partir de ellas, es posible reflexionar y revisar el propio quehacer, resignificarlo, elaborar nuevas propuestas, plasmarlas en el aula y evaluar su implementación.

Hay temas fundamentales que podrían incluirse en espacios formativos para los docentes del programa que surgen del estudio realizado, tales como a) la tutoría como dispositivo pedagógico de orientación y acompañamiento; b) las competencias comunicativas en relación con objetivos, metodología y evaluación de las cátedras; c) las prácticas de lectura y escritura como procesos epistémicos; d) la elaboración de consignas para distintas instancias de evaluación; e) la mirada experta del docente sobre las producciones escritas de los estudiantes y las devoluciones que se realizan; y f) la elaboración de grillas de evaluación en relación con las competencias comunicativas.

Otra línea de trabajo que puede ser un aporte al programa es la realización de espacios de reflexión conjunta entre los tutores y la coordinación para elaborar una revisión crítica de los motivos que subyacen a su accionar, y la correlación que existe entre ellos y los objetivos que el programa ha explicitado. De esta manera, se podrían evidenciar teorías implícitas que orientan las actividades que se presentan desde el programa, en aras de propiciar cambios que permitan construir propuestas tendientes a desarrollar mejores aprendizajes en los estudiantes. 
Los procesos de reflexión podrían extenderse a encuentros entre la coordinación del programa y los directores de los departamentos de las carreras. Dichos encuentros podrían orientarse a desarrollar la noción de competencias comunicativas y la necesidad de que más grupos institucionales estén involucrados en los procesos de enseñanza que atraviesan toda la formación universitaria de los profesionales ingenieros.

A su vez, se abren perspectivas para desarrollar estudios comparados con programas de tutoría similares en otras sedes de la Universidad Tecnológica Nacional, así como en otras universidades de este país y casas de estudio de otros países. Además, en trabajos futuros, será relevante desarrollar modelos de tutoría en competencias de lectura y escritura desde un enfoque sociocultural.

Los resultados del análisis contribuyen con la construcción de conocimiento de la tutoría como política y como práctica institucional, pues impacta en los trayectos académicos de los estudiantes, y media sobre su inclusión, permanencia y egreso. Asimismo, se abren perspectivas para considerar la redefinición de las prácticas docentes. De igual manera, a partir del estudio, emergen posibles implicancias para el desarrollo de políticas curriculares en la educación superior que incluyan las prácticas de lectura y escritura al interior de las disciplinas, desde el ingreso y a lo largo de todas las carreras universitarias, abordadas desde perspectivas socioculturales. En este sentido, aparece, también, la posibilidad de impactar en la generación de políticas que favorezcan el desarrollo y la formación profesional docente en relación con una didáctica sociocultural de la lectura y la escritura académica. Estas propuestas se enmarcan en políticas inclusivas y universales que promueven el desarrollo integral de todos los sujetos, a través de la igualdad de oportunidades educativas. 


\section{Referencias bibliográficas}

Baldoni, M., Barrón, M., Corrado, R., Eizaguirre, M., Fernández, G., Goñi, M., Izuzquiza, M., Laxalt, I. y Tomelliri, M. (2005). Las tutorías de estudio. Reflexiones de un proyecto en marcha en la Facultad de Ciencias Humanas de la Universidad Nacional del Centro de la Provincia de Buenos Aires. Trabajo presentado en I Congreso Nacional de la Sociedad Argentina de Estudios Comparados en Educación (pp. 1-13), Buenos Aires. Recuperado de http://www.saece.org.ar/docs/congresol/Baldoni.doc

Baquero, R. (2001). La educabilidad bajo sospecha. Cuaderno de Pedagogía, 9 , 71-85.

(2006). Del individuo auxiliado al sujeto en situación: Algunos problemas en los usos de los enfoques socioculturales en educación. Revista Espacios en Blanco, 16, 123-151.

Bixio, B. (2003). Pasos hacia una didáctica sociocultural de la lengua y la literatura: Sociolingüística y educación, un campo tensionado. Lulú Coquette. Revista de Didáctica de la Lengua y la Literatura, 2, 24-35.

Bombini, G. (2005). Una mirada transversal para pensar las relaciones entre lenguaje y escuela, Revista Quaderns Digitals. Recuperado 9 de marzo de 2016 de: http://www.quadernsdigitals.net/index.php?accionMenu=hemeroteca. VisualizaArticuloIV.visualiza\&articulo id $=8790$

(2009). La inclusión educativa en las zonas de pasaje: representaciones y prácticas de lectura y escritura. En E. Martos, T. Rösing (Coords.) Prácticas de Lectura y Escritura (pp. 431-444). Passo Fundo: UPF Editora.

Bombini, G. y Cuesta, C. (2006). Lengua y literatura: Campo de la didáctica específica y prácticas de enseñanza. En G. Fioriti (Comp.), Didácticas específicas. Reflexiones y aportes para la enseñanza (pp. 53-77). Buenos Aires: Miño y Dávila.

Cadzen, C. (1991). El discurso en el aula. El lenguaje de la enseñanza y del aprendizaje. Barcelona: Paidós.

Capelari, M. (2009). Las configuraciones del rol del tutor en la universidad argentina: Aportes para reflexionar acerca de los significados que se construyen sobre el fracaso educativo en la educación superior. Revista Iberoamericana de Educación 49(8), 1-10.

-_-_- (2016). El rol del tutor en la universidad. Configuraciones, significados y prácticas. Buenos Aires: Sb editorial.

Carlino, P. (2005). Escribir, leer y aprender en la universidad. Buenos Aires: Fondo de Cultura Económica.

- - _- (2013). Alfabetización académica diez años después. Revista Mexicana de Investigación Educativa, 18, (57), 355-381.

28 I Castronovo, A. y Mancovsky, V. (2010). La lectura y la escritura como procesos centrales de los aprendizajes disciplinares: La inclusión y la pertenencia a 
una comunidad de lectura específica. En A. Vázquez, M. Novo, I. Jakob y L. Pelliza (Comps.), Lectura, escritura y aprendizaje disciplinar. (pp. 819828). Río Cuarto: UniRío.

Charlot, B. (2008). La relación con el saber. Elementos para una teoría. Buenos Aires: Libros del Zorzal.

Clérici, J. y Amieva, R. (2013). Las perspectivas de docentes ingenieros sobre las tutorías en la universidad. Revista de Docencia Universitaria, 11, 375-395.

Desinano, N. (2009). Los alumnos universitarios y la escritura académica. Análisis de un problema. Rosario: Homo Sapiens Ediciones.

Díaz, C. Stangatti, F. y Festa, C. (2012). Tutorías. Acompañar y contener para leer y escribir. En L. Laco, L. Natale y M. Ávila (Comps.), La lectura y la escritura en la formación académica, docente y profesional. Buenos Aires: edUTecNe.

Elissetche, G. (2010). La cátedra compartida como espacio de problematización de las prácticas de lectura y escritura en la universidad. Nuestra experiencia en la UNLa. En A. Vázquez, M. Novo, I. Jakob y L. Pelliza (Comps.), Lectura, escritura y aprendizaje disciplinar (pp. 489-492). Río Cuarto: UniRío.

Estienne, V. (2012). Leer y escribir en la universidad. Propuesta de intervención para los primeros años. En L. Laco, L. Natale y M. Ávila. (Comps.), La lectura y la escritura en la formación académica, docente y profesional. Buenos Aires: edUTecNe.

Fenstermarcher, G. (1989). La investigación de la enseñanza: Enfoques, teorías $y$ métodos. Barcelona: Paidós.

Garbarini, M., Escobar, M., Lavigna, L. y López D’ Amato, S. (2016). Programa para el Fortalecimiento de la Lectura y la Escritura. Experiencias TAPTA en materias del ámbito científico - UNAJ. Trabajo presentado en V Jornadas Nacionales y I Latinoamericanas de Ingreso y Permanencia en Carreras Científico-Tecnológicas (pp. 141- 146). Universidad Tecnológica Nacional. Facultad Regional Bahía Blanca. Recuperado de http://redipecyt.fceia. unr.edu.ar/trabajos/33-IPECyT-2016.pdf

García, V., Nícoli, E. y Urús, M. (2012). Tutorías Académicas en Competencias de Lectura y Escritura. En L. Laco, L. Natale y M. Ávila. (Comps.), La lectura y la escritura en la formación académica, docente y profesional. Buenos Aires: edUTecNe.

Gutiérrez, K. y Rogoff, B. (2003). Cultural Ways of Learning: Individual Traits or Repertoires of Practice, Educational Researcher, 32(5), 19-25. Recuperado 12 de abril de 2016 de: $\underline{\text { http://er.aera.net }}$

Laco, L. y Ávila, M. (2012). Una experiencia en alfabetización académica: evolución, análisis y resultados. Buenos Aires: Universidad Tecnológica Nacional. Facultad Regional General Pacheco.

Laco, L. y Guiggiani, L. (2008). Programa Institucional de Tutorías. Un modelo integral. Buenos Aires: Universidad Tecnológica Nacional. Facultad Regional General Pacheco. 
Litwin, E. (1996) El campo de la didáctica: La búsqueda de una nueva agenda. En A. W. de Camilloni, M. C. Davini, G. Edestein, E. Litwin, M. Souto, S. Barco (Eds.) Corrientes didácticas contemporáneas (pp. 91-115). Buenos Aires: Paidós.

-_-_ (1997). Las configuraciones didácticas. Una nueva agenda para la educación Superior. Buenos Aires: Paidós.

Mercer, N. (1997) La construcción guiada del conocimiento. El habla de profesores y alumnos. Madrid: Paidós.

Moreno, L. (2010). Acción docente y acción tutorial ¿Una misma función? En P. Ducoing (Coord.), Tutoría y Mediación II (pp. 95-112). México: Iisue Educación.

Murillo, J. (2012). Programa para el Centro de Apoyo para la lectura, la oralidad y la escritura - DIGA. En L. Laco, L. Natale y M. Ávila. (Comps.), La lectura y la escritura en la formación académica, docente y profesional. (s.p.) Buenos Aires: edUTecNe.

Natale, L. y Stagnaro, D. (2013). Desarrollo de habilidades de lectura y escritura en la trayectoria académica del ingeniero: La experiencia de un programa desafiante e innovador. Revista Argentina de Enseñanza de la Ingeniería, 3, 45-52. Recuperado de http://www.ing.unrc.edu.ar/raei/archivos/img/ arc 2013-03-19 $21 \quad 06$ 03-N3T04.pdf

Rigonat, M. y García, C. (2012). La escritura académica en la formación de grado. Reflexiones a partir de la puesta en práctica de talleres específicos tutoriales. En L. Laco, L. Natale y M. Ávila. (Comps.), La lectura y la escritura en la formación académica, docente y profesional. Buenos Aires: edUTecNe.

Rogoff, B. (1993). Aprendices del pensamiento. El desarrollo cognitivo en el contexto social. Barcelona: Paidós.

Urús, M. (2017). Prácticas de escritura de docentes ingenieros: la relación con el saber. En G. Bombini y P. Labeur. (Comps.), Leer y escribir en las zonas de pasaje. Articulaciones entre la escuela secundaria y el nivel superior (pp. 95-104). Buenos Aires: Biblos.

Zavala, V. (2009). “¿Quién está diciendo eso?” Literacidad académica, Identidad y poder en la Educación Superior. En J. Kalman, B. Street (Coords.), Lectura, escritura y matemáticas como prácticas sociales: Diálogos con América Latina (pp. 348-363). México D. F.: Siglo XXI.

Zerillo, A. (2014). Trabajo colaborativo de lectoescritura académica en el contexto de las disciplinas. Trabajo presentado en I Jornada de Investigación Interdepartamental, Universidad Nacional de La Matanza. Recuperado de http://studylib.es/doc/606893/trabajo-colaborativo-de-lectoescrituraacad\%C3\%A9mica-en-el 